\title{
Novel and preclinical treatment strategies in pneumococcal meningitis
}

\author{
Jan P. Bewersdorf ${ }^{\mathrm{a}}$, Denis Grandgirard ${ }^{\mathrm{b}}$, Uwe Koedel ${ }^{\mathrm{a}}$, and Stephen L. Leib ${ }^{\mathrm{b}}$
}

\begin{abstract}
Purpose of review
Pneumococcal meningitis is the most frequent form of bacterial meningitis in Europe and the United States. Although early antimicrobial and adjuvant therapy with dexamethasone have helped to improve disease outcome in adults, mortality and morbidity rates remain unsatisfactorily high, emphasizing the need for additional treatment options. Promising targets for adjuvant therapy have been identified recently and will be the focus of this review.
\end{abstract}

\begin{abstract}
Recent findings
Brain disease in pneumococcal meningitis is caused by direct bacterial toxicity and excessive meningeal inflammation. Accordingly, promising targets for adjuvant therapy comprise limiting the release of toxic bacterial products and suppressing inflammation in a way that maximally protects against tissue injury without hampering pathogen eradication by antibiotics. Among the agents tested so far in experimental models, complement inhibitors, matrix-metalloproteinase inhibitors, and nonbacteriolytic antibiotics or a combination of the above have the potential to more efficiently protect the brain either alone (e.g., in children and outside the high-income settings) or in addition to adjuvant dexamethasone. Additionally, new protein-based pneumococcal vaccines are being developed that promise to improve disease prevention, namely by addressing the increasing problem of serotype replacement seen with pneumococcal conjugate vaccines.
\end{abstract}

\section{Summary}

Pneumococcal meningitis remains a life-threatening disease requiring early antibiotic and targeted antiinflammatory therapy. New adjuvant therapies showed promising results in animal models but need systematic clinical testing.

\section{Keywords}

adjuvant therapy, management, pneumococcal meningitis, pneumococcal vaccine

\section{INTRODUCTION}

The implementation of pneumococcal conjugate vaccine programs has reduced the incidence of invasive pneumococcal diseases in many countries, albeit with varying degrees of success. The latter might be related to intercountry differences in vaccination coverage, serotype distribution, and most importantly for the future, serotype replacement. Notwithstanding, meningitis caused by Streptococcus pneumoniae, a Gram-positive encapsulated bacterium, remains the most common form of community-acquired bacterial meningitis in adults, at least in the Western world $\left[1^{\mathbf{*}}, 2,3^{\mathbf{*}}\right]$. Although an improved case management system has been shown to be effective in ameliorating disease outcome $\left[2,4,5,6^{\mathbf{*}}\right]$, the reported mortality rates still range between 5 and $18 \%$ even in an advanced Western hospital setting $\left[1^{\mathbf{*}}, 2,7,8^{\mathbf{*}}\right]$, but are substantially higher reaching over $30 \%$ when considered globally
$[2,9]$. Among surviving patients, up to $50 \%$ suffer from long-term neurologic sequelae such as hearing loss, seizures, or neurocognitive dysfunction $\left[4,8^{-}, 10\right]$.

Over the past decade, experimental work has provided evidence that death and sequelae because of pneumococcal meningitis occur as a consequence of a hyperinflammatory host response to the pathogen [11]. Accordingly, adjuvant therapy with

aDepartment of Neurology, Klinikum Grosshadern, Ludwig Maximilians-
Universität München, Munich, Germany and b Institute for Infectious
Diseases, University of Bern, Bern, Switzerland
Correspondence to Prof. Dr. Uwe Koedel, Department of Neurology,
Ludwig Maximilians-Universität München, Marchioninistrasse 15, D-
81377 Munich, Germany. Tel: +4989440073144 ;
fax: +49894400 75561; e-mail: Uwe.Koedel@med.uni-muenchen.de Curr Opin Infect Dis 2017, 30:000-000

DOI:10.1097/QC0.0000000000000416 


\section{KEY POINTS}

- Several adjuvant therapies to attenuate the injurious proinflammatory reaction in pneumococcal meningitis have been identified in animal experiments but have not been tested in human patients yet.

- Inhibition of the complement system, reduction of the release of proinflammatory bacterial components by using nonlytic antimicrobials, and prevention of IFN- $\gamma$ and MMP-mediated inflammatory processes including BBB breakdown seem to be promising candidates for adjuvant therapy.

- Protein-based pneumococcal vaccines are currently being studied in early phase clinical trials and promise to provide broader coverage than current conjugate pneumococcal vaccines.

- Emerging antimicrobial-resistant S. pneumoniae strains have been isolated necessitating close surveillance and the development of new antibiotics.

dexamethasone has been found to significantly reduce mortality and sequelae from pneumococcal meningitis in adults $[12,13]$ and is currently recommended in the American and European guidelines for adults with suspected pneumococcal meningitis. For potential benefit, dexamethasone must be given before or concomitantly with the antibiotics $\left[3^{-\prime}, 14\right]$. Of note, the beneficial effects of dexamethasone only apply to adult patients with pneumococcal meningitis in high-income countries [13]. Although to date no differences on long-term neuropsychological sequelae were reported in meningitis patients treated with dexamethasone compared with placebo, adjunctive dexamethasone has been reported to negatively affect survival and hippocampal neuroregeneration in experimental meningitis $\left[15^{\prime}, 16\right]$. The effect of dexamethasone indicates that immunomodulatory treatments (might) have the potential to improve the outcome of pneumococcal meningitis. Its limitations in applicability and also efficacy underline the need for novel adjunctive medications. Several interesting candidates have been identified in recent studies. The purpose of this review is to provide a brief overview of our current understanding of the pathogenesis of pneumococcal meningitis and its potential implications on the management of this life-threatening disease in humans.

\section{PATHOGENESIS OF PNEUMOCOCCAL MENINGITIS}

The principal ecological niche of $S$. pneumoniae is the human nasopharynx. To successfully invade the central nervous system (CNS), it must overcome many obstacles on its journey through the blood to the brain. The CNS is protected externally by its covering leptomeninges and internally (against blood-borne pathogen invasion) by the blood-brain barrier (BBB) and the blood-cerebrospinal (CSF) barrier (BCSFB). To get access to the CNS, the successful pathogen therefore requires a defect in the external barrier (e.g., a posttraumatic dura leak). It also uses a multistep process involving the colonization of the nasopharyngeal mucosa, followed by the invasion into the blood with successful survival and replication, and finally the traversal of the $\mathrm{BBB}[17,18]$.

\section{PREVENTION OF BACTERIAL TRAVERSAL OF THE BLOOD-BRAIN BARRIER}

The BBB is formed by brain endothelial cells, astrocytes, and pericytes to protect the CNS from invading pathogens [19]. It has recently been shown that $S$. pneumoniae that were able to enter the CNS expressed adhesive rlrA-regulated gene A (RrgA)-containing pili [20]. Using brain biopsies from patients who died of pneumococcal meningitis and a murine meningitis model, S. pneumoniae was further found to colocalize with two BBB endothelial receptors, namely polymeric immunoglobulin receptor (pIgR) and platelet endothelial cell adhesion molecule (PECAM-1). Thereby, the bacteria-receptor interaction is predominantly mediated by the above-mentioned pilus protein RrgA [21"',22]. Blockade of pIgR and PECAM1 with targeted antibodies substantially reduced bacterial invasion of the subarachnoid space in a bacteremia-derived meningitis model [21"'] (Fig. 1). More interestingly, these antibodies further decreased bacterial CNS load in mice cotreated with ceftriaxone, the first-line empiric antimicrobial recommended for community-acquired bacterial meningitis in humans $\left[3^{-"}, 21^{-"}\right]$. Therefore, blockade of pIgR and PECAM-1 could be an interesting target for adjuvant therapy of pneumococcal meningitis, namely by shielding the brain against pneumococcal (re)entry. Not only the prevention of pneumococcal (re)entry but also the preservation of $\mathrm{BBB}$ integrity has been shown to be beneficial during bacterial meningitis. Matrix metalloproteinases (MMPs) participate in the disruption of the BBB by degrading the extracellular matrix, and their pharmacological inhibition reduced BBB permeability and improved the outcome (mortality and neuronal damage) in different experimental models $[11,23-26]$.

\section{TARGETED IMMUNOMODULATION}

Excessive inflammation significantly contributes to meningitis-associated brain damage and thus an 


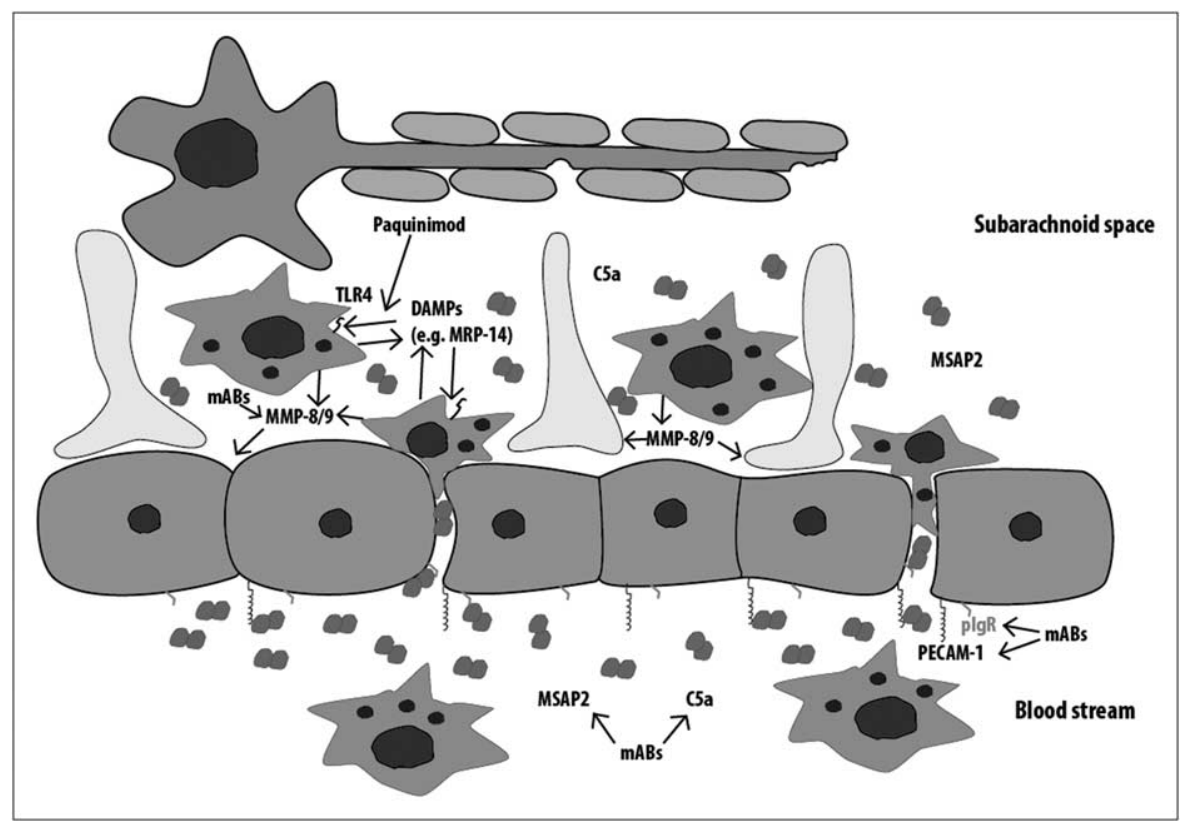

FIGURE 1. Selected pathogenetic aspects of pneumococcal meningitis and potential therapeutic targets. BBB, blood-brain barrier; CNS, central nervous system; DAMP, danger-associated molecular pattern; IFN, interferon; $m A B$, monoclonal antibodies; MASP, mannose-binding lectin-associated serine protease; MMP, matrix metalloproteinase; MRP, myeloid-related protein; PECAM, platelet endothelial cell adhesion molecule; plgR, polymeric immunoglobulin receptor; TLR, toll-like receptor.

unfavorable disease outcome [27-31]. This hyperinflammatory reaction is related to the host's initial incompetence to handle pneumococcal infection in the CSF, because of a lack of innate immune agents including complement and other opsonins. Thus, $S$. pneumoniae can proliferate almost unrestrained and large quantities of immune-stimulatory pathogenassociated molecular patterns can be released into the CSF, resulting in a fulminant inflammatory reaction $\left[30^{*}\right]$. This resulting hyperinflammation causes collateral tissue damage with release of endogenous danger-associated molecular patterns (DAMPs) which in turn can propagate and aggravate inflammation. Therefore, several promising candidates for new adjuvant therapies act by modulating this detrimental hyperinflammatory reaction via various mechanisms (Fig. 1).

Fast and efficient bactericidal antimicrobial substances that do not cause bacteriolysis prevent the release of bacterial components and dampen the inflammatory burst after initiation of antimicrobial therapy. Daptomycin, which has been successfully used in individual case reports of meningitis because of Gram-positive drug-resistant bacteria, fulfills these characteristics [32-36]. In these studies, daptomycin given intravenously was found to penetrate into the CSF at approximatively $0.8-5 \%$ of the serum concentration $(11.5 \%$ when corrected for protein binding) [34,36-39]. Compared with ceftriaxone it more rapidly cleared $S$. pneumoniae from the
CSF, decreased the levels of proinflammatory cytokines in the CSF, and was associated with less neuronal injury and improved outcome in several experimental studies of pneumococcal meningitis $[40,41]$. Given the increasing prevalence of penicillin-resistant $S$. pneumoniae strains as well as the emergence of ceftriaxone and fluoroquinoloneresistant isolates, new antibiotics such as daptomycin provide an additional therapeutic option for severe or antibiotic-resistant cases [42-46]. However, daptomycin acts on Gram-positive pathogens only and, thus, needs to be combined with a broadspectrum antibiotic for empirical therapy [41].

The complement cascade is activated via three different pathways with the classic complement path being the most important one for invasive pneumococcal infection [47]. All pathways converge not only to mediate opsonization of bacteria for phagocytosis and/or direct cell lysis via the membrane-attack complex but also to enhance inflammation through the production of anaphylatoxins like complement component 5a (C5a) [27]. Pathogenic pneumococcal strains have developed several virulence factors such as surface receptors, a polysaccharide capsule, and pneumolysin to counteract complement-mediated clearance from the blood stream $\left[27,48,49,50^{\circ}\right]$. Acquired or congenital defects of the complement system are associated with a higher susceptibility to and worse outcomes of bacterial meningitis $[27,51-53]$. On the other 
hand, genetic deficiency and/or pharmacologic inhibition of complement factors like complement components 1q (C1q), 3 (C3), 5 (C5) and mannosebinding lectin-associated serine protease (MASP) 2 were highly effective in suppressing the CNS inflammatory response to pneumococcal infection [54"',55-57] (Fig. 1). Accordingly, an association between elevated levels of various complement factors and a more severe disease course and worse prognosis were demonstrated in CSF samples from patients with pneumococcal meningitis [58]. CSF concentrations of complement factors and their clinical correlations clearly differ between pneumococcal and meningococcal meningitis [58]. In pneumococcal meningitis, high CSF complement levels were a strong indicator of disease severity and mortality, whereas an inverse relationship between terminal complement complex-9 levels and mortality is observed in meningococcal meningitis [58]. Accordingly, complement activation by application of recombinant properdin afforded protection against meningococcal sepsis and reduced the severity of pneumococcal infection by enhancing bacterial killing [59]. These data highlight different roles of the complement system in the host defense against major meningeal pathogens. Therapeutic inhibition of the complement system needs, therefore, to be highly specific by preventing uncontrolled immune activation while preserving complement-mediated bacterial killing as demonstrated with specific C5a neutralization in a human whole blood model of meningococcal sepsis [60].

Another potential new target might be the inhibition of the proinflammatory mediator, macrophage migration inhibitory factor (MIF). MIF is a central modulator of the innate immune system and increased levels are associated with an adverse outcome in severe bacterial infections including pneumococcal pneumonia and meningitis [28",61-64]. Additionally, inhibition of MIF signaling has been shown to ameliorate disease outcomes in animal models of pneumococcal and Escherichia coli sepsis $[65,66]$.

Recent experimental studies have also suggested involvement of the proinflammatory cytokine interferon (IFN)- $\gamma$ in pneumococcal meningitis, which is released mainly by natural killer cells via an inflammasome-induced, interleukin 18-mediated mechanism $[67,68]$. In murine pneumococcal meningitis models, IFN- $\gamma$ has been shown to be a key modulator of proinflammatory cytokine production via the induction of nitric oxide synthase 2 and to thereby mediate breakdown of the BBB and ultimately impact disease outcome [67-70]. Accordingly, antibody-mediated inhibition as well as genetic deficiency of IFN- $\gamma$ or components of the interleukin 18-inflammasome pathway induced a strong protective effect in murine models of pneumococcal meningitis $[67,68,71]$. Importantly, these effects of IFN- $\gamma$ were reproduced in different clinically pathogenic $S$. pneumoniae strains making attenuation of the IFN- $\gamma$ mediated hyperinflammatory response another promising target for adjuvant therapy [68].

Several MMPs not only mediate brain damage by degrading the extracellular matrix and increasing BBB permeability but also participate in the modulation of inflammation by virtue of their sheddase activity. For example, a disintegrin and metallopeptidase (ADAM) domain 17, also known as tumor necrosis factor- $\alpha$ converting enzyme (TACE), processes precursor tumor necrosis factor into a soluble, more active form which contributes to the spread of inflammation. TACE, therefore, contributes to the hyperinflammatory pathophysiology of bacterial meningitis. In several experimental studies, MMP inhibitors with TACE inhibiting activity attenuated CSF inflammation in pneumococcal meningitis, reduced brain damage and improved survival rate and neurofunctional outcome rendering them highly attractive candidates for adjuvant therapy [11,24] (Fig. 1). Moreover, combining the effects of MMP inhibitors with another modulator of the inflammatory reaction, such as daptomycin may increase the beneficial effects (Muri L, Grandgirard D, Leib SL, unpublished data).

Massive inflammation is only little altered by appropriate antibiotic therapy over days even though complete CSF sterilization occurs within hours. Inflammation-induced cell injury leads to the release of DAMPs that further sustain inflammation and exacerbate damage [31,72]. Among the DAMPs involved in this process are the myeloidrelated protein (MRP) 14 and high-mobility group box-1 protein. When injected intrathecally, MRP14 was sufficient to induce meningitis in mice by activating toll-like receptor 4 and both MRP14 deficiency and direct inhibition of MRP14-toll-like receptor 4 interaction with paquinimod attenuated disease outcome [31,73] (Fig. 1). Although treatment with high-mobility group box-1 protein inhibitors had no effect on the development of meningitis, it led to a better resolution of inflammation during antibiotic therapy, which was accompanied by reduced brain disease and better disease outcome [72].

In summary, several interesting candidates for immunomodulatory adjunct therapy have been identified recently. Among these, highly specific complement system inhibitors, MMP/TACE inhibitors as well as nonbacteriolytic antibiotics such as daptomycin seem to be the most promising ones in 
Table 1. Mechanisms of action and stage of development of potential adjuvant therapies

\begin{tabular}{|c|c|c|c|}
\hline & Mechanisms of action & Stage of development & References \\
\hline Paquinimod & $\begin{array}{l}\text { Direct and specific inhibition of } \\
\text { MRP14-TLR4 interaction }\end{array}$ & Cell culture and rodent experiments & [31] \\
\hline Anti-MIF antibody & $\begin{array}{l}\text { Attenuation of excess inflammatory } \\
\text { response of innate immune system }\end{array}$ & $\begin{array}{l}\text { Cell culture and rodent experiments } \\
\text { Antibody used in phase I clinical } \\
\text { study for other indication }\end{array}$ & {$[28 *, 56-62]$} \\
\hline Anti-IFN- $\gamma$ antagonists & $\begin{array}{l}\text { Antibody-mediated inhibition of IFN- } \gamma \\
\text { prevents BBB breakdown and } \\
\text { reduces production of } \\
\text { proinflammatory cytokines }\end{array}$ & Cell culture and rodent experiments & {$[63-68]$} \\
\hline $\begin{array}{l}\text { Specific complement } \\
\text { inhibitors }\end{array}$ & $\begin{array}{l}\text { Blockade of complement factor } \mathrm{C} 5 \mathrm{a} \\
\text { or MASP2 }\end{array}$ & Cell culture and rodent experiments & $\begin{array}{l}{[54=, 55,47,50 *} \\
\quad 51-53]\end{array}$ \\
\hline Daptomycin & $\begin{array}{l}\text { Attenuation of antibiotic-mediated } \\
\text { hyperinflammation }\end{array}$ & $\begin{array}{l}\text { Rodent experiments and sporadic } \\
\text { use in clinics }\end{array}$ & {$[31-33,35-41]$} \\
\hline MMP/TACE inhibitors & $\begin{array}{l}\text { Inhibition of BBB disruption and/or } \\
\text { soluble cytokine release }\end{array}$ & Cell culture and rodent experiments & {$[11,24,26,36]$} \\
\hline
\end{tabular}

$\mathrm{BBB}$, blood-brain barrier; IFN, interferon; MASP, mannose-binding lectin-associated serine protease; MIF, migration inhibitory factor; MMP, matrix metalloproteinase; MRP, myeloid-related protein; TACE, tumor necrosis factor- $\alpha$ converting enzyme; TLR, toll-like receptor.

ameliorating disease severity and reducing mortality in rodent experimental models. These findings strengthen the hypothesis that a large extent of neuronal injury in pneumococcal meningitis is not caused by toxic effects from $S$. pneumoniae itself but is rather mediated by the hyperinflammatory response of the host's immune system. No clinical studies have been performed in humans thus far and it remains to be elucidated whether these promising results can be reproduced across species. Table 1 summarizes the mechanism of action and stage of development of potential immunomodulating adjuvant therapies.

\section{NEURODEGENERATION AND REGENERATION: ADDITIONAL THERAPEUTIC TARGETS?}

Histopathological studies document a wide spectrum of brain injury associated with pneumococcal meningitis in humans, including cortical petechial hemorrhages, focal necrotic lesions in cortical and subcortical structures, and loss of myelinated fibers in the white matter [74"-]. Additionally, mild-tomoderate apoptosis of granular cells in the dentate gyrus was found in more than two-thirds of cases in a previous autoptic study [75]. More recently, however, no significant differences in the number of apoptotic cells were detectable between meningitis cases and control patients [74"']. In animal studies, the occurrence and degree of neuronal apoptosis depend on multiple factors, such as the age, strain, and species of the animal used as well as the causative pathogen. Although previous research focused primarily on strategies to prevent neurodegeneration, recent work also investigated neuroregenerative processes following meningitis.

Neurotrophic factors are key mediators of neuronal survival and neurogenesis. Amongst them, brain-derived neurotrophic factor (BDNF) is up regulated during pneumococcal meningitis in both mice and humans via activation of the myeloid differentiation primary response 88 (MyD88)/ nuclear factor kappa B (NF-kB) signaling pathway, a classical pathway of the innate immune system involved in the pathogenesis of pneumococcal meningitis [76-78]. Activation of this pathway, therefore, seems to be essential to counter $S$. pneumoniae infection but to also protect neurons from excess injury and initiate functional recovery. As dexamethasone has been shown to attenuate MyD88/ NF-кB signaling [79], this link between neuroregeneration and immune response might also explain why dexamethasone increased neuronal apoptosis and impaired neuroregeneration in the hippocampus in an infant rat model of pneumococcal meningitis $[14,80]$. Conversely, treatment with exogenous BDNF decreased hippocampal apoptosis and stimulated neurogenesis and differentiation of neural progenitor cells in the hippocampus following pneumococcal meningitis in rodent models $\left[81^{-"}, 82,83\right]$. However, whether these new neurons can integrate into neuronal circuits and facilitate functional recovery remains to be elucidated.

\section{FUTURE PERSPECTIVES}

One of the major causes for the reduced incidence of pneumococcal meningitis has been the introduction and widespread use of pneumococcal conjugate 
vaccines [2]. The reduction in incidence is most prominent for pneumococcal serotypes included in pediatric conjugate vaccines but an increase in invasive pneumococcal infections caused by nonvaccine serotypes is observed $\left[1^{*}, 2,84-86\right]$. This serotype replacement is of clinical importance as it has been shown that the $S$. pneumoniae capsule type has a direct effect on disease severity [50"]. Alternative pneumococcal vaccines using highly conserved protein antigens such as pneumococcal histidine triad protein D and pneumolysin are currently in development and have been shown to have an acceptable safety and reactogenicity profile in phase I/II studies. These novel vaccines promise to provide broader coverage than the current conjugate vaccines and would thereby eliminate the problem of serotype replacement [87-89].

Although primary prophylaxis through widespread vaccination should remain the primary goal in the future, we should also remain vigilant to the emergence of multidrug-resistant $S$. pneumoniae strains $[45,46,90,91]$. Therefore, the development and clinical testing of novel antimicrobials is highly warranted. The ideal profile of potential agents is a good BBB penetration and a rapid bactericidal activity with limited release of bacterial structures causing the inflammatory burst associated with an adverse outcome [40]. Also clinical trials investigating the combination of nonlytic antibiotics such as daptomycin, as well as MMP/TACE and complement system inhibitors may yield promising results (Muri L, Grandgirard D, Leib SL, unpublished data).

\section{CONCLUSION}

Although the incidence and mortality from pneumococcal meningitis have declined over recent years, several challenges remain. Pneumococcal meningitis still has a mortality rate of up to $18 \%$ and a substantial proportion of surviving patients sustains debilitating neurologic sequelae. Even as new protein-based pneumococcal vaccines promise a broader coverage of pneumococcal serotypes, further systematic research on adjuvant therapy and new antimicrobials is highly warranted. Promising targets focus on modulating the excessive immune response that has been repeatedly linked to worse clinical outcomes. Potential new therapeutic options include nonlytic antimicrobials such as daptomycin, highly specific complement system inhibitors, and exogenous neurotrophic factors. Optimally, modulating the immune response should preserve its ability to eradicate the pathogen, should limit the extent and duration of the hyperinflammation causing damage, and should favor the initiation of regenerative processes.

\section{Acknowledgements}

The work is a collaborative project from the European Society of Clinical Microbiology and Infectious Diseases (ESCMID) Study Group for Infectious Diseases of the Brain (ESGIB).

\section{Financial support and sponsorship}

The work was supported by the German Research Foundation (to UK: KO1974/5-1,6-1,7-1), the Else KrönerFresenius Stiftung (to UK: 2013_A239), and the Swiss National research Foundation (to SLL: 310030_ 162583).

\section{Conflicts of interest}

There are no conflicts of interest.

\section{REFERENCES AND RECOMMENDED \\ READINC}

Papers of particular interest, published within the annual period of review, have been highlighted as:

- of special interest

- of outstanding interest

1. Bijlsma MW, Brouwer MC, Kasanmoentalib ES, et al. Community-acquired

- bacterial meningitis in adults in the Netherlands, 2006-14: a prospective cohort study. Lancet Infect Dis 2016; 16:339-347.

The large cohort study shows the effect of pneumococcal conjugate vaccine and improved clinical management of pneumococcal meningitis on disease epidemiology and case fatality rate.

2. McGill F, Heyderman RS, Panagiotou $S$, et al. Acute bacterial meningitis in adults. Lancet 2016; 388:3036-3047.

3. van de Beek D, Cabellos C, Dzupova O, et al., ESCMID Study Group for

I. Infections of the Brain (ESGIB). ESCMID guideline: diagnosis and treatment of acute bacterial meningitis. Clin Microbiol Infect 2016; 22(Suppl 3):S37-S62.

Current European guidelines on the diagnosis and treatment of bacterial meningitis.

4. Dorsett M, Liang SY. Diagnosis and treatment of central nervous system infections in the emergency department. Emerg Med Clin North Am 2016; 34:917-942.

5. Bodilsen J, Brandt CT, Sharew A, et al. Early versus late diagnosis in community-acquired bacterial meningitis: a retrospective cohort study. Clin Microbiol Infect 2017.

6. Bodilsen J, Dalager-Pedersen M, Schonheyder HC, Nielsen H. Time to

- antibiotic therapy and outcome in bacterial meningitis: a Danish population-based cohort study. BMC Infect Dis 2016; 16:392.

The authors provide evidence that a delay in initiating antimicrobial therapy is associated with a higher mortality which has important implications on clinical practice.

7. Hasbun R, Rosenthal N, Balada-Llasat JM, et al. Epidemiology of meningitis and encephalitis in the United States, 2011-2014. Clin Infect Dis 2017; 65:359-363.

8. Buchholz G, Koedel U, Pfister HW, et al. Dramatic reduction of mortality in - pneumococcal meningitis. Crit Care 2016; 20:312.

The study shows a remarkable reduction in mortality to about $5 \%$ from bacterial meningitis, thanks to early initiation of empiric antimicrobials, adjuvant dexamethasone, and improvements of neurocritical care of associated complications. However, among the survivors up to $50 \%$ suffer from some degree of neurologic complications.

9. GBD 2013 Mortality and Causes of Death Collaborators. Global, regional, and national age-sex specific all-cause and cause-specific mortality for 240 causes of death, 1990-2013: a systematic analysis for the Global Burden of Disease Study 2013. Lancet 2015; 385:117-171.

10. Lucas MJ, Brouwer MC, van de Beek D. Neurological sequelae of bacterial meningitis. J Infect 2016; 73:18-27.

11. Meli DN, Coimbra RS, Erhart DG, et al. Doxycycline reduces mortality and injury to the brain and cochlea in experimental pneumococcal meningitis. Infect Immun 2006; 74:3890-3896.

12. de Gans J, van de Beek D; European Dexamethasone in Adulthood Bacterial Meningitis Study Investigators. Dexamethasone in adults with bacterial meningitis. N Engl J Med 2002; 347:1549-1556. 
13. Brouwer MC, Mclntyre $P$, Prasad K, van de Beek D. Corticosteroids for acute bacterial meningitis. Cochrane Database Syst Rev 2015; (9):CD004405.

14. Tunkel AR, Hartman BJ, Kaplan SL, et al. Practice guidelines for the management of bacterial meningitis. Clin Infect Dis 2004; 39:1267-1284.

15. Bally L, Grandgirard D, Leib SL. Inhibition of hippocampal regeneration by adjuvant dexamethasone in experimental infant rat pneumococcal meningitis. Antimicrob Agents Chemother 2016; 60:1841-1846.

The study shows that adjuvant therapy with dexamethasone can impair neuronal regeneration in mice supporting rationale for investigating new adjuvant therapies apart from dexamethasone.

16. Spreer A, Gerber J, Hanssen M, et al. Dexamethasone increases hippocampal neuronal apoptosis in a rabbit model of Escherichia coli meningitis. Pediatr Res 2006; 60:210-215

17. Doran KS, Fulde M, Gratz N, et al. Host-pathogen interactions in bacterial meningitis. Acta Neuropathol 2016; 131:185-209.

18. lovino F, Seinen J, Henriques-Normark B, van Dijl JM. How does streptococcus pneumoniae invade the brain? Trends Microbiol 2016; 24:307-315.

19. Kim KS. Mechanisms of microbial traversal of the blood-brain barrier. Nat Rev Microbiol 2008; 6:625-634.

20. lovino F, Hammarlof DL, Garriss G, et al. Pneumococcal meningitis is promoted by single cocci expressing pilus adhesin RrgA. J Clin Invest 2016; 126:2821-2826.

21. lovino $F$, Engelen-Lee JY, Brouwer $M$, et al. plgR and PECAM-1 bind to

- pneumococcal adhesins RrgA and PspC mediating bacterial brain invasion. J Exp Med 2017; 214:1619-1630.

The experimental study provides insight into the pathogenesis of CNS invasion by

S. pneumoniae and identifies potential new therapeutic targets.

22. Iovino F, Molema G, Bijlsma JJ. Platelet endothelial cell adhesion molecule-1, a putative receptor for the adhesion of Streptococcus pneumoniae to the vascular endothelium of the blood-brain barrier. Infect Immun 2014; 82:3555-3566.

23. Liechti FD, Bachtold F, Grandgirard D, et al. The matrix metalloproteinase inhibitor RS-130830 attenuates brain injury in experimental pneumococcal meningitis. J Neuroinflammation 2015; 12:43.

24. Liechti FD, Grandgirard D, Leppert D, Leib SL. Matrix metalloproteinase inhibition lowers mortality and brain injury in experimental pneumococcal meningitis. Infect Immun 2014; 82:1710-1718.

25. Ricci S, Grandgirard D, Wenzel M, et al. Inhibition of matrix metalloproteinases attenuates brain damage in experimental meningococcal meningitis. BMC Infect Dis 2014; 14:726.

26. Sellner J, Leib SL. In bacterial meningitis cortical brain damage is associated with changes in parenchymal MMP-9/TIMP-1 ratio and increased collagen type IV degradation. Neurobiol Dis 2006; 21:647-656.

27. Mook-Kanamori BB, Geldhoff $M$, van der Poll T, van de Beek D. Pathogenesis and pathophysiology of pneumococcal meningitis. Clin Microbiol Rev 2011; 24:557-591.

28. Savva A, Brouwer MC, Roger $T$, et al. Functional polymorphisms of macro-

- phage migration inhibitory factor as predictors of morbidity and mortality of pneumococcal meningitis. Proc Natl Acad Sci U S A 2016; 113:3597-3602.

The study identified genetic variations in a proinflammatory mediator (MIF) in human patients and linked it to disease outcome. Additionally, the authors could show that the inhibition of MIF can ameliorate pneumococcal meningitis in a mouse model.

29. Zierhut M, Dyckhoff $S$, Masouris I, et al. Role of purinergic signaling in experimental pneumococcal meningitis. Sci Rep 2017; 7:44625.

30. van de Beek D, Brouwer M, Hasbun R, et al. Community-acquired bacterial - meningitis. Nat Rev Dis Primers 2016; 2:16074.

Comprehensive review on epidemiology, pathogenesis, and management of community-acquired bacterial meningitis.

31. Wache C, Klein M, Ostergaard C, et al. Myeloid-related protein 14 promotes inflammation and injury in meningitis. J Infect Dis 2015; 212:247-257.

32. Denetclaw TH, Suehiro I, Wang PK, Tolliver GL. Successful treatment of ventriculostomy-associated meningitis caused by multidrug resistant coagulase-negative Staphylococcus epidermidis using low-volume intrathecal daptomycin and loading strategy. Ann Pharmacother 2014; 48:1376-1379.

33. Kelesidis $\mathrm{T}$, Humphries $\mathrm{R}$, Ward $\mathrm{K}$, et al. Combination therapy with daptomycin, linezolid, and rifampin as treatment option for MRSA meningitis and bacteremia. Diagn Microbiol Infect Dis 2011; 71:286-290.

34. Le J, Bookstaver PB, Rudisill CN, et al. Treatment of meningitis caused by vancomycin-resistant Enterococcus faecium: high-dose and combination daptomycin therapy. Ann Pharmacother 2010; 44:2001-2006.

35. Erritouni M, Ktaich N, Rahal JJ, et al. Use of daptomycin for the treatment of methicillin-resistant coagulase-negative staphylococcal ventriculitis. Case Rep Med 2012; 2012:593578.

36. Vena A, Falcone M, Comandini $\mathrm{E}$, et al. Daptomycin plus trimethoprim/ sulfamethoxazole combination therapy in postneurosurgical meningitis caused by linezolid-resistant Staphylococcus epidermidis. Diagn Microbiol Infect Dis 2013; 76:99-102.

37. Riser MS, Bland CM, Rudisill CN, Bookstaver PB. Cerebrospinal fluid penetration of high-dose daptomycin in suspected Staphylococcus aureus meningitis. Ann Pharmacother 2010; 44:1832-1835.

38. Vivas M, Force E, Garrigos C, et al. Experimental study of the efficacy of daptomycin for the treatment of cephalosporin-resistant pneumococcal meningitis. J Antimicrob Chemother 2014; 69:3020-3026.
39. Kullar R, Chin JN, Edwards DJ, et al. Pharmacokinetics of single-dose daptomycin in patients with suspected or confirmed neurological infections. Antimicrob Agents Chemother 2011; 55:3505-3509.

40. Grandgirard D, Schurch $C$, Cottagnoud $P$, Leib SL. Prevention of brain injury by the nonbacteriolytic antibiotic daptomycin in experimental pneumococcal meningitis. Antimicrob Agents Chemother 2007; 51:2173-2178.

41. Grandgirard D, Burri M, Agyeman $P$, Leib SL. Adjunctive daptomycin attenuates brain damage and hearing loss more efficiently than rifampin in infant rat pneumococcal meningitis. Antimicrob Agents Chemother 2012; 56: $4289-4295$

42. Brouwer MC, Tunkel AR, van de Beek D. Epidemiology, diagnosis, and antimicrobial treatment of acute bacterial meningitis. Clin Microbiol Rev 2010; 23:467-492.

43. Whitney CG, Farley MM, Hadler J, et al., Active Bacterial Core Surveillance Program of the Emerging Infections Program Network. Increasing prevalence of multidrug-resistant Streptococcus pneumoniae in the United States. N Engl J Med 2000; 343:1917-1924.

44. Cottagnoud $P$, Pfister $M$, Acosta $F$, et al. Daptomycin is highly efficacious against penicillin-resistant and penicillin and quinolone-resistant pneumococ$\mathrm{ci}$ in experimental meningitis. Antimicrob Agents Chemother 2004; 48: 3928-3933.

45. Kim $\mathrm{SH}$, Song $\mathrm{JH}$, Chung $\mathrm{DR}$, et al. Changing trends in antimicrobial resistance and serotypes of Streptococcus pneumoniae isolates in Asian countries: an Asian Network for Surveillance of Resistant Pathogens (ANSORP) study. Antimicrob Agents Chemother 2012; 56:1418-1426.

46. Metcalf BJ, Chochua S, Gertz RE Jr, et al. Using whole genome sequencing to identify resistance determinants and predict antimicrobial resistance phenotypes for year 2015 invasive pneumococcal disease isolates recovered in the United States. Clin Microbiol Infect 2016; 22:1002.e1-1002.e8.

47. Brown JS, Hussell T, Gilliland SM, et al. The classical pathway is the dominant complement pathway required for innate immunity to Streptococcus pneumoniae infection in mice. Proc Natl Acad Sci U S A 2002; 99:16969-16974.

48. Li J, Glover DT, Szalai AJ, et al. PspA and PspC minimize immune adherence and transfer of pneumococci from erythrocytes to macrophages through their effects on complement activation. Infect Immun 2007; 75:5877-5885

49. Kadioglu A, Weiser JN, Paton JC, Andrew PW. The role of Streptococcus pneumoniae virulence factors in host respiratory colonization and disease. Nat Rev Microbiol 2008; 6:288-301.

50. Hathaway $\sqcup$, Grandgirard D, Valente LG, et al. Streptococcus pneumoniae

- capsule determines disease severity in experimental pneumococcal meningitis. Open Biol 2016; 6:

The study could show that $S$. pneumoniae capsular subtype has an impact on disease severity and outcome which is of clinical relevance as some degree of serotype replacement has been observed following the widespread use of pneumococcal conjugate vaccines.

51. Adriani KS, Brouwer MC, Geldhoff M, et al. Common polymorphisms in the complement system and susceptiblity to bacterial meningitis. J Infect 2013; 66:255-262.

52. Brouwer MC, de Gans J, Heckenberg SG, et al. Host genetic susceptibility to pneumococcal and meningococcal disease: a systematic review and metaanalysis. Lancet Infect Dis 2009; 9:31-44.

53. Davila S, Wright VJ, Khor CC, et al., International Meningococcal Genetics Consortium. Genome-wide association study identifies variants in the CFH region associated with host susceptibility to meningococcal disease. Nat Genet 2010; 42:772-776.

54. Kasanmoentalib ES, Valls Seron M, Ferwerda B, et al. Mannose-binding

- lectin-associated serine protease 2 (MASP-2) contributes to poor disease outcome in humans and mice with pneumococcal meningitis. J Neuroinflammation 2017; 14:2

The study provides additional evidence that very specific inhibition of components of the complement system can ameliorate pneumococcal meningitis in mice.

55. Kasanmoentalib ES, Valls Seron M, Morgan BP, et al. Adjuvant treatment with dexamethasone plus anti-C5 antibodies improves outcome of experimental pneumococcal meningitis: a randomized controlled trial. J Neuroinflammation 2015; 12:149.

56. Rupprecht TA, Angele B, Klein M, et al. Complement $\mathrm{C} 1 \mathrm{q}$ and $\mathrm{C} 3$ are critical for the innate immune response to Streptococcus pneumoniae in the central nervous system. J Immunol 2007; 178:1861-1869.

57. Woehrl B, Brouwer MC, Murr C, et al. Complement component 5 contributes to poor disease outcome in humans and mice with pneumococcal meningitis. J Clin Invest 2011; 121:3943-3953.

58. Mook-Kanamori BB, Brouwer MC, Geldhoff M, et al. Cerebrospinal fluid complement activation in patients with pneumococcal and meningococcal meningitis. J Infect 2014; 68:542-547.

59. Ali YM, Hayat A, Saeed BM, et al. Low-dose recombinant properdin provides substantial protection against Streptococcus pneumoniae and Neisseria meningitidis infection. Proc Natl Acad Sci U S A 2014; 111: $5301-5306$

60. Sprong $\mathrm{T}$, Brandtzaeg $\mathrm{P}$, Fung $\mathrm{M}$, et al. Inhibition of $\mathrm{C} 5 \mathrm{a}$-induced inflammation with preserved C5b-9-mediated bactericidal activity in a human whole blood model of meningococcal sepsis. Blood 2003; 102:3702-3710.

61. Calandra $T$, Roger $T$. Macrophage migration inhibitory factor: a regulator of innate immunity. Nat Rev Immunol 2003; 3:791-800. 
62. Das R, Subrahmanyan L, Yang IV, et al. Functional polymorphisms in the gene encoding macrophage migration inhibitory factor are associated with Gram-negative bacteremia in older adults. J Infect Dis 2014; 209: $764-768$

63. Roger T, David J, Glauser MP, Calandra T. MIF regulates innate immune responses through modulation of toll-like receptor 4. Nature 2001; 414:920-924

64. Doernberg S, Schaaf B, Dalhoff K, et al. Association of macrophage migration inhibitory factor (MIF) polymorphisms with risk of meningitis from Streptococcus pneumoniae. Cytokine 2011; 53:292-294.

65. Roger T, Schneider A, Weier M, et al. High expression levels of macrophage migration inhibitory factor sustain the innate immune responses of neonates. Proc Natl Acad Sci U S A 2016; 113:E997-E1005

66. Weiser JN, Roche AM, Hergott CB, et al. Macrophage migration inhibitory factor is detrimental in pneumococcal pneumonia and a target for therapeutic immunomodulation. J Infect Dis 2015; 212:1677-1682.

67. Mitchell AJ, Yau B, McQuillan JA, et al. Inflammasome-dependent IFN- $\gamma$ drives pathogenesis in Streptococcus pneumoniae meningitis. J Immunol 2012; 189:4970-4980.

68. Yau B, Too LK, Ball HJ, Hunt NH. TIGR4 strain causes more severe disease than WU2 strain in a mouse model of Streptococcus pneumoniae meningitis: a common pathogenic role for interferon- $\gamma$. Microbes Infect 2017; 19:413-421.

69. Too LK, Ball HJ, McGregor IS, Hunt NH. The pro-inflammatory cytokine interferon-gamma is an important driver of neuropathology and behavioural sequelae in experimental pneumococcal meningitis. Brain Behav Immun $2014 ; 40: 252-268$

70. Yau B, Mitchell AJ, Too LK, et al. Interferon- $\gamma$-induced nitric oxide synthase-2 contributes to blood/brain barrier dysfunction and acute mortality in experimental streptococcus pneumoniae meningitis. J Interferon Cytokine Res 2016; 36:86-99.

71. Pettini E, Fiorino F, Cuppone AM, et al. Interferon- $\gamma$ from brain leukocytes enhances meningitis by type 4 Streptococcus pneumoniae. Front Microbiol 2015; 6:1340.

72. Hohne $C$, Wenzel M, Angele B, et al. High mobility group box 1 prolongs inflammation and worsens disease in pneumococcal meningitis. Brain 2013; 136:1746-1759.

73. Vogl T, Tenbrock K, Ludwig S, et al. Mrp8 and Mrp14 are endogenous activators of toll-like receptor 4 , promoting lethal, endotoxin-induced shock. Nat Med 2007; 13:1042-1049.

74. Engelen-Lee JY, Brouwer MC, Aronica E, van de Beek D. Pneumococcal

-1. meningitis: clinical-pathological correlations (MeninGene-Path). Acta Neuropathol Commun 2016; 4:26.

Large study on brain autopsies from patients who died from pneumococcal meningitis. The authors derived a neuropathological disease severity score based on extent of inflammation and vascular injury.

75. Nau R, Soto A, Bruck W. Apoptosis of neurons in the dentate gyrus in humans suffering from bacterial meningitis. J Neuropathol Exp Neurol 1999; 58: $265-274$.
76. Tauber SC, Stadelmann C, Spreer A, et al. Increased expression of BDNF and proliferation of dentate granule cells after bacterial meningitis. J Neuropathol Exp Neurol 2005; 64:806-815.

77. Gerber J, Tauber SC, Armbrecht I, et al. Increased neuronal proliferation in human bacterial meningitis. Neurology 2009; 73:1026-1032.

78. $\mathrm{Xu} \mathrm{D}$, Lian $\mathrm{D}$, Zhang $Z$, et al. Brain-derived neurotrophic factor is regulated via MyD88/NF-kB signaling in experimental Streptococcus pneumoniae meningitis. Sci Rep 2017; 7:3545.

79. Chen M, Reed RR, Lane AP. Acute inflammation regulates neuroregeneration through the NF-kB pathway in olfactory epithelium. Proc Natl Acad Sci U S A $2017 ; 114: 8089-8094$.

80. Leib SL, Heimgartner C, Bifrare YD, et al. Dexamethasone aggravates hippocampal apoptosis and learning deficiency in pneumococcal meningitis in infant rats. Pediatr Res 2003; 54:353-357.

81. Lian $\mathrm{D}, \mathrm{He} \mathrm{D}, \mathrm{Wu} \mathrm{J}$, et al. Exogenous BDNF increases neurogenesis in the

I. hippocampus in experimental Streptococcus pneumoniae meningitis. J Neuroimmunol 2016; 294:46-55.

The study identified BDNF as a potential adjunct therapy in pneumococcal meningitis as it stimulates proliferation of neuronal progenitor cells in the hippocampus.

82. Xu D, Lian D, Wu J, et al. Brain-derived neurotrophic factor reduces inflammation and hippocampal apoptosis in experimental Streptococcus pneumoniae meningitis. J Neuroinflammation 2017; 14:156.

83. Bifrare YD, Kummer J, Joss $P$, et al. Brain-derived neurotrophic factor protects against multiple forms of brain injury in bacterial meningitis. J Infect Dis 2005; 191:40-45.

84. Harboe ZB, Dalby T, Weinberger DM, et al. Impact of 13-valent pneumococcal conjugate vaccination in invasive pneumococcal disease incidence and mortality. Clin Infect Dis 2014; 59:1066-1073.

85. Tin Tin Htar M, Christopoulou D, Schmitt HJ. Pneumococcal serotype evolution in Western Europe. BMC Infect Dis 2015; 15:419.

86. Waight PA, Andrews NJ, Ladhani SN, et al. Effect of the 13-valent pneumococcal conjugate vaccine on invasive pneumococcal disease in England and Wales 4 years after its introduction: an observational cohort study. Lancet Infect Dis 2015; 15:535-543.

87. Leroux-Roels G, Maes C, De Boever F, et al. Safety, reactogenicity and immunogenicity of a novel pneumococcal protein-based vaccine in adults: a phase I/II randomized clinical study. Vaccine 2014; 32:6838-6846.

88. Leroux-Roels I, Devaster JM, Leroux-Roels G, et al. Adjuvant system AS02V enhances humoral and cellular immune responses to pneumococcal protein PhtD vaccine in healthy young and older adults: randomised, controlled trials. Vaccine 2015; 33:577-584.

89. Pichichero ME, Khan MN, Xu Q. Next generation protein based Streptococcus pneumoniae vaccines. Hum Vaccin Immunother 2016; 12:194-205.

90. van de Beek D, Brouwer MC, Thwaites GE, Tunkel AR. Advances in treatment of bacterial meningitis. Lancet 2012; 380:1693-1702.

91. Abdelkader MM, Aboshanab KM, El-Ashry MA, Aboulwafa MM. Prevalence of MDR pathogens of bacterial meningitis in Egypt and new synergistic antibiotic combinations. PLoS One 2017; 12:e0171349. 